\title{
Angular Ligand Constraint Yields an Improved Olefin Aziridination Catalyst
}

\author{
Andrei N. Vedernikov* and Kenneth G. Caulton*
}

\section{Supplemental information}

\section{Experimental}

General. All manipulations were carried out under purified argon using standard Schlenk and glove box techniques. Solvents were dried and distilled following standard protocols and stored in gastight bulbs under argon. All reagents for which a synthesis is not given are commercially available from Aldrich and were used as received without further purification. All NMR solvents were dried, vacuumtransferred and stored in an argon-filled glovebox. [2.1.1]-(2,6)-pyridinophane ${ }^{1}$ and 2,6-bis(2pyridylmethyl)pyridine (tpdm), (tpdm) $\mathrm{CuCl}^{2}$ were synthesized according to the published procedures.

${ }^{1} \mathrm{H}$ and ${ }^{13} \mathrm{C}$ NMR spectra were recorded on Inova 700 spectrometer $\left({ }^{1} \mathrm{H} 400 \mathrm{MHz} ;{ }^{13} \mathrm{C} 100.62\right.$

$\mathrm{MHz}) .{ }^{1} \mathrm{H}$ and ${ }^{13} \mathrm{C}$ NMR chemical shifts are reported in ppm and referenced to residual solvent resonance peaks.

Computational details. Theoretical calculations in this work have been performed using density functional theory (DFT) method, ${ }^{3}$ specifically functional $\mathrm{PBE},{ }^{4}$ implemented in an original program package "Priroda". In PBE calculations relativistic Stevens-Basch-Krauss (SBK) effective core potentials (ECP) ${ }^{6}$ optimized for DFT-calculations have been used. The basis set was 311-split for main group elements with one additional polarization p-function for hydrogen, additional two polarization dfunctions for elements of higher periods. Full geometry optimization has been performed without constraints on symmetry. For all species under investigation frequency analysis has been carried out. All minima have been checked for the absence of imaginary frequencies.

LCuCl. Optimized geometry with selected bond distances $(\AA)$ and angles (deg.) (hydrogen atoms are omitted for clarity): 


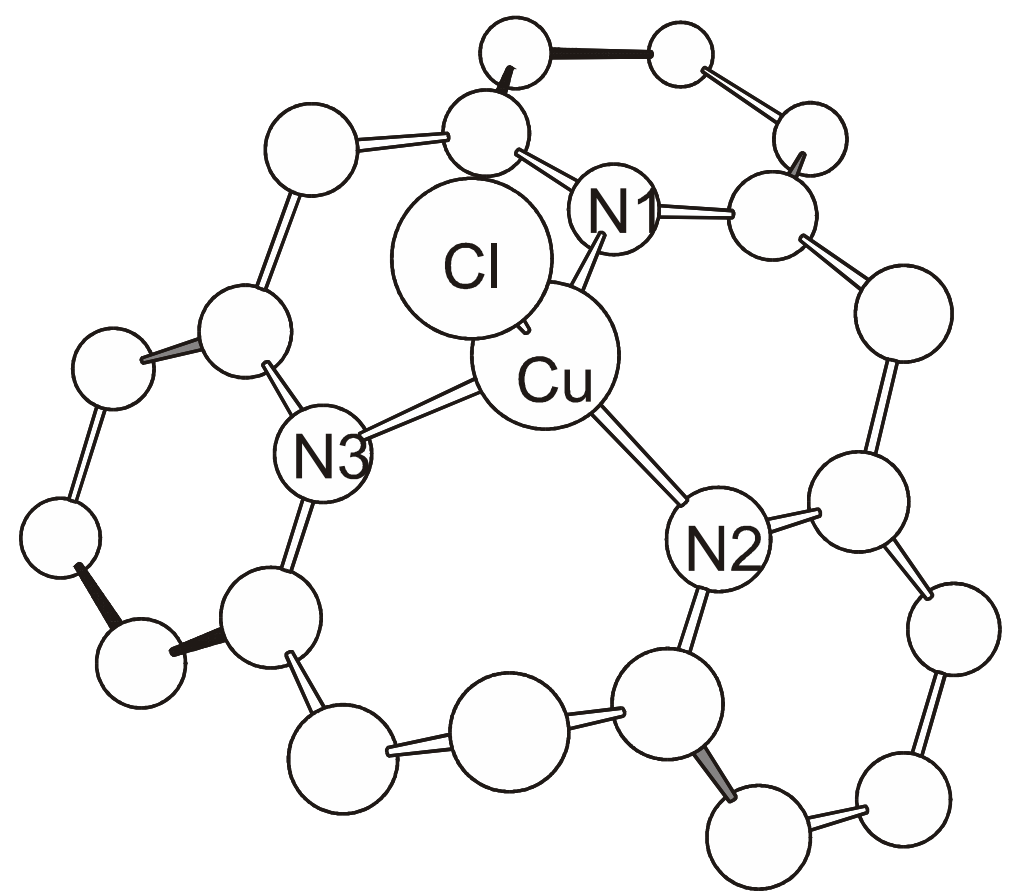

$\mathrm{Cu}-\mathrm{N}(1), 2.037 ; \mathrm{Cu}-\mathrm{N}(2), 2.035 ; \mathrm{Cu}-\mathrm{N}(3), 2.180 ; \mathrm{Cu}-\mathrm{Cl}, 2.220 ; \mathrm{Cl}-\mathrm{Cu}-\mathrm{N}(1), 125.8 ; \mathrm{Cl}-\mathrm{Cu}-\mathrm{N}(2)$, $128.5 ; \mathrm{N}(1)-\mathrm{Cu}-\mathrm{N}(2), 92.4 ; \mathrm{Cl}-\mathrm{Cu}-\mathrm{N}(3), 110.6$.

$\mathbf{L C u}^{+}$. Optimized geometry with selected bond distances $(\AA)$ and angles (deg.) (hydrogen atoms are omitted for clarity):

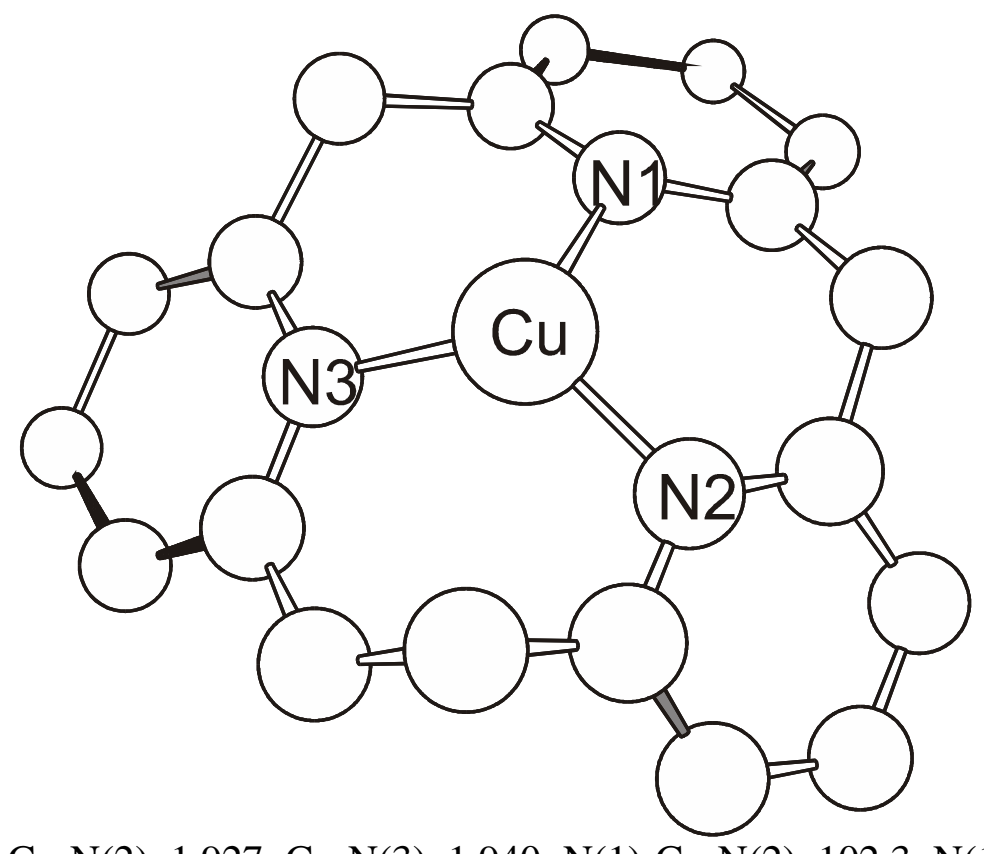

$\mathrm{Cu}-\mathrm{N}(1), 1.975 ; \mathrm{Cu}-\mathrm{N}(2), 1.927 ; \mathrm{Cu}-\mathrm{N}(3), 1.940 ; \mathrm{N}(1)-\mathrm{Cu}-\mathrm{N}(2), 102.3 ; \mathrm{N}(1)-\mathrm{Cu}-\mathrm{N}(3), 104.7$; $\mathrm{N}(2)-\mathrm{Cu}-\mathrm{N}(3), 122.7$.

LCu(NTs) ${ }^{2+}$. Spin population: copper, 6\%; nitrene nitrogen, $76 \%$; oxygen atoms, $18 \%$. 
LCuCl, $\mathbf{C}_{19} \mathbf{H}_{17} \mathrm{ClN}_{3} \mathbf{C u}$. To a dry flask containing a magnetic stirring bar was added, in a glove box, $\mathrm{CuCl}(99 \mathrm{mg}, 1.0 \mathrm{mmol})$ and $20.0 \mathrm{~mL}$ of dry benzene. To the stirred mixture, [2.1.1]-(2,6)pyridinophane $(287 \mathrm{mg}, 1.0 \mathrm{mmol})$ dissolved in $5.0 \mathrm{~mL}$ of benzene was added. Stirring was continued at room temperature for $5 \mathrm{~h}$. The orange precipitate formed was filtered off, washed twice with $1.0 \mathrm{~mL}$ portions of benzene and dried. Yield $370 \mathrm{mg}(95 \%)$.

${ }^{1} \mathrm{H}$ NMR $\left(\mathrm{CD}_{2} \mathrm{Cl}_{2}, 22^{\circ} \mathrm{C}\right), 3.25\left(\mathrm{~m}, 2 \mathrm{H}, \mathrm{C}_{2} \mathrm{H}_{4}\right), 4.11\left(\mathrm{~d}, J=13.0 \mathrm{~Hz}, 2 \mathrm{H}, \mathrm{CH}_{2}\right), 4.38\left(\mathrm{~m}, 2 \mathrm{H}, \mathrm{C}_{2} \mathrm{H}_{4}\right)$, $4.71\left(\mathrm{~d}, J=13.0 \mathrm{~Hz}, 2 \mathrm{H}, \mathrm{CH}_{2}\right), 7.10(\mathrm{~d}, J=8.0 \mathrm{~Hz}, 2 \mathrm{H}$, meta-CH), $7.12(\mathrm{~d}, J=7.8 \mathrm{~Hz}, 2 \mathrm{H}$, meta-CH), $7.25(\mathrm{~d}, J=7.8 \mathrm{~Hz}, 2 \mathrm{H}$, meta-CH), $7.50(\mathrm{t}, J=7.8 \mathrm{~Hz}, 2 \mathrm{H}$, para-CH), $7.60(\mathrm{t}, J=7.8 \mathrm{~Hz}, 1 \mathrm{H}$, para-CH).

${ }^{13} \mathrm{C}$ NMR $\left(\mathrm{CD}_{2} \mathrm{Cl}_{2},-45^{\circ} \mathrm{C}\right), 35.91\left(\mathrm{CH}_{2}\right), 46.54\left(\mathrm{C}_{2} \mathrm{H}_{4}\right), 122.03,122.26,123.05$ (meta-C, py), 137.25, 137.87 (para-C, py), 155.02, 155.51, 159.70 (ortho-C, py).

$\mathbf{L C u C l}_{2}, \mathbf{C}_{\mathbf{1 9}} \mathbf{H}_{17} \mathrm{Cl}_{2} \mathbf{N}_{\mathbf{3}} \mathbf{C u}$. To a dry flask containing a magnetic stirring bar was added, in a glove box, $\mathrm{CuCl}_{2}(135 \mathrm{mg}, 1.0 \mathrm{mmol})$ and $20.0 \mathrm{~mL}$ of dry benzene. To the stirred mixture, [2.1.1]-(2,6)pyridinophane $(287 \mathrm{mg}, 1.0 \mathrm{mmol}$ ) dissolved in $5.0 \mathrm{~mL}$ of benzene was added. Stirring was continued at room temperature for $5 \mathrm{~h}$. The green precipitate formed was filtered off, washed twice with $1.0 \mathrm{~mL}$ portions of benzene and dried. Yield $400 \mathrm{mg}(95 \%)$.

$\mathrm{LCu}(\mathrm{OTf})_{2}, \mathrm{C}_{21} \mathrm{H}_{17} \mathrm{~F}_{6} \mathbf{N}_{3} \mathrm{O}_{6} \mathrm{~S}_{2} \mathrm{Cu}$. To a dry flask containing a magnetic stirring bar was added, in a glove box, $\mathrm{Cu}(\mathrm{OTf})_{2}(361 \mathrm{mg}, 1.0 \mathrm{mmol})$ and $20.0 \mathrm{~mL}$ of dry benzene. To the stirred mixture, [2.1.1](2,6)-pyridinophane $(287 \mathrm{mg}, 1.0 \mathrm{mmol}$ ) dissolved in $5.0 \mathrm{~mL}$ of benzene was added. Stirring was continued at room temperature for $5 \mathrm{~h}$. The light green precipitate formed was filtered off, washed twice with $1.0 \mathrm{~mL}$ portions of benzene and dried. Yield $600 \mathrm{mg}(95 \%)$.

“Activation" of pre-catalysts $\mathrm{LCuCl}_{n}$ with $\mathrm{NaBAr}_{4}(\mathrm{Ar}=\mathrm{Ph}, 3,5$-bis(trifluoromethyl)phenyl). In an argon-filled glovebox a $5 \mathrm{ml}$ Schlenk flask equipped with a Teflon valve and a magnetic stirring bar was charged with $10 \mu \mathrm{mol}$ of $\mathrm{LCuCl}_{\mathrm{n}}, 20(\mathrm{n}=2)$ or $10(\mathrm{n}=1) \mu \mathrm{mol}$ of $\mathrm{NaBAr}_{4}$ and $0.6 \mathrm{ml}$ of $\mathrm{CD}_{2} \mathrm{Cl}_{2}$. In one minute large chunks of $\mathrm{NaBAr}_{4}{ }_{4}$ dissolved, new fine precipitate appeared and the liquid color turned lightyellow. According to NMR data, in the case of $\mathrm{LCuCl}$ all starting complex was consumed suggesting that the yield of $\mathrm{LCuBAr}_{4}^{\mathrm{F}}$ is almost quantitative. Catalytic activity of this solution remained unchanged after two days at r.t.

LCuBAr ${ }_{4}{ }_{4}{ }^{1} \mathrm{H}$ NMR $\left(\mathrm{CD}_{2} \mathrm{Cl}_{2},-45^{\circ} \mathrm{C}\right), 3.32\left(\mathrm{~m}, 2 \mathrm{H}, \mathrm{C}_{2} \mathrm{H}_{4}\right), 3.91\left(\mathrm{~m}, 2 \mathrm{H}, \mathrm{C}_{2} \mathrm{H}_{4}\right), 4.16(\mathrm{~d}, J=13.8$ $\left.\mathrm{Hz}, 2 \mathrm{H}, \mathrm{CH}_{2}\right), 4.24\left(\mathrm{~d}, J=13.8 \mathrm{~Hz}, 2 \mathrm{H}, \mathrm{CH}_{2}\right), 7.17(\mathrm{~d}, J=7.8 \mathrm{~Hz}, 2 \mathrm{H}$, meta-CH), $7.22(\mathrm{~d}, J=7.8 \mathrm{~Hz}, 2 \mathrm{H}$, meta-CH), $7.32\left(\mathrm{~d}, J=7.8 \mathrm{~Hz}, 2 \mathrm{H}\right.$, meta-CH), $7.52\left(\mathrm{br} \mathrm{s}, 4 \mathrm{H}\right.$, para- $\left.\mathrm{CH}, \mathrm{BAr}_{4}^{\mathrm{F}}\right), 7.57(\mathrm{t}, J=7.8 \mathrm{~Hz}, 2 \mathrm{H}$, para- $\mathrm{CH}), 7.66\left(\mathrm{t}, J=7.8 \mathrm{~Hz}, 1 \mathrm{H}\right.$, para-CH), $7.71\left(\mathrm{br} \mathrm{s}, 8 \mathrm{H}\right.$, ortho- $\left.\mathrm{CH}, \mathrm{BAr}_{4}{ }_{4}\right)$.

${ }^{13} \mathrm{C}$ NMR $\left(\mathrm{CD}_{2} \mathrm{Cl}_{2},-45^{\circ} \mathrm{C}\right), 35.97\left(\mathrm{CH}_{2}\right), 46.55\left(\mathrm{C}_{2} \mathrm{H}_{4}\right), 117.73\left(\mathrm{~m}\right.$, para-C, $\left.\mathrm{BAr}_{4}^{\mathrm{F}}\right), 123.02,123.28$, 123.97 (meta-C, py), 124.66 (quart, ${ }^{1} J_{\mathrm{C}-\mathrm{F}}=272.7 \mathrm{~Hz}, \mathrm{CF}_{3}, \mathrm{BAr}{ }_{4}{ }^{\mathrm{F}}$ ), 128.88 (quart, ${ }^{2} J_{\mathrm{C}-\mathrm{F}}=31.7 \mathrm{~Hz}$, meta-C, $\mathrm{BAr}_{4}^{\mathrm{F}}$ ), 134.89 (br s, ortho-C, $\mathrm{BAr}_{4}^{\mathrm{F}}$ ), 138.89, 139.63 (para-C, py), 155.12, 155.73, 159.64 (ortho-C, py), $161.92\left(\right.$ quart, ${ }^{1} J_{\mathrm{B}-\mathrm{C}}=50 \mathrm{~Hz}, \mathrm{~B}-\mathrm{C}, \mathrm{BAr}^{\mathrm{F}}{ }_{4}$ ).

In the case of copper(II) complex ( $\mathrm{n}=2$ ), catalytic activity was completely lost in $1 \mathrm{~h}$.

Catalytic olefin aziridination with PhINTs. The flask with solution of activated catalyst was charged with 1-5 equ. of olefin (liquids), removed from glove box and cooled in an ice or acetone - liquid nitrogen bath if necessary. In the case of ethylene, propylene, cis-2-butene and 1-butene, the necessary amount (1-10 equ.) of gas taken at $1 \mathrm{~atm}$ and $20^{\circ} \mathrm{C}$ was condensed. Then the mixture was allowed to warm up to $0^{\circ}$ or r.t. and 1 equiv. of PhINTs was added in one portion. Immediate red color appeared. In a few minutes, the precipitate of PhINTs dissolved and the contents of the flask was transferred to a Teflon- 
capped NMR tube. The solution color persisted or turned green indicating, in the last case, catalyst degradation.

Yield of corresponding aziridine(s) and product of $\alpha$-amination was determined by NMR integration relative to signals of unreacted olefin (liquids) and / or PhI (gases). The two values differed from one another by no more than 4-8\%. Concentration of gaseous substrates in solution was determined by integration of their signals relative those of $\mathrm{BAr}_{4}$.

Aziridine formed was isolated by filtering the resulting solution through a $2 \mathrm{~cm}$ layer of alumina put in a micro-column with subsequent washing with dichloromethane. The filtrate was dried under vacuum to remove solvent and $\mathrm{PhI}$ and weighted. NMR of the residue was taken to assure in the identity and purity of the product.

Spectral patterns of aziridines and $\alpha$-tosylaminoolefins obtained were identical to those reported in literature: N-tosylaziridine, ${ }^{7}$ 2-methyl-N-tosylaziridine ${ }^{8}$ 2-ethyl-N-tosylaziridine, ${ }^{9}$ 2-tert-butyl-Ntosylaziridine, ${ }^{10}$ 2,2,3,3-tetramethyl-N-tosylaziridine, ${ }^{11}$ 2-carbomethoxy-N-tosylaziridine, ${ }^{12} 2$ carbomethoxy-2-methyl-N-tosylaziridine, ${ }^{13} \mathrm{~N}$-tosyl-6-azabicyclo[3.1.0]hexane, ${ }^{14} \mathrm{~N}$-tosyl-7azabicyclo[4.1.0] heptane, N-tosyl-9-azabicyclo[6.1.0]nonane, ${ }^{15} \mathrm{~N}$-(cyclopent-2-enyl)tosylamide, $\mathrm{N}$ (cyclohex-2-enyl)tosylamide, ${ }^{16} \mathrm{~N}$-(1-methyl-2-propenyl)tosylamide. ${ }^{17}$

cis-2,3-Dimethyl-N-tosylaziridine. ${ }^{18}$ Major component of a mixture resulting from aziridination of cis-2-butene. ${ }^{1} \mathrm{H}$ NMR $\left(\mathrm{CDCl}_{3}, 22^{\circ} \mathrm{C}\right), \delta: 1.15\left(\mathrm{vd},{ }^{3} J_{\mathrm{H}-\mathrm{H}}=5.0 \mathrm{~Hz}, 6 \mathrm{H}, \mathrm{Me}\right.$, azir), $2.41(\mathrm{~s}, 3 \mathrm{H}, \mathrm{Me}, \mathrm{Tol})$, $2.84\left(\mathrm{~m}, 2 \mathrm{H}, \mathrm{CH}\right.$, azir), $7.30\left(\mathrm{~d},{ }^{3} J_{\mathrm{H}-\mathrm{H}} 8.0 \mathrm{~Hz}, \mathrm{CH}, \mathrm{Tol}\right), 7.78\left(\mathrm{~d},{ }^{3} J_{\mathrm{H}-\mathrm{H}} 8.0 \mathrm{~Hz}, \mathrm{CH}, \mathrm{Tol}\right) ;{ }^{13} \mathrm{C} \mathrm{NMR}\left(\mathrm{CDCl}_{3}\right.$, $22^{\circ} \mathrm{C}$ ), $\delta: 11.85$ (Me, azir), $21.80(\mathrm{Me}, \mathrm{Tol}), 40.48$ (CH, azir), 127.81, 129.85 (ortho-, meta-C, Tol), 135.85, 144.39 (ipso-, para-C, Tol).

trans-2,3-Dimethyl-N-tosylaziridine. ${ }^{18}$ Minor component of a mixture resulting from aziridination of $c i s$-2-butene. ${ }^{1} \mathrm{H}$ NMR $\left(\mathrm{CDCl}_{3}, 22{ }^{\circ} \mathrm{C}\right), \delta:{ }^{1} \mathrm{H} \mathrm{NMR}\left(\mathrm{CDCl}_{3}, 22{ }^{\circ} \mathrm{C}\right), \delta: 1.40\left(\mathrm{~d},{ }^{3} J_{\mathrm{H}-\mathrm{H}}=5.0\right.$ $\mathrm{Hz}, 6 \mathrm{H}, \mathrm{Me}$, azir), 2.40 (s, 3H, Me, Tol), 2.70 (m, 2H, CH, azir), 7.30 (d, $\left.{ }^{3} J_{\mathrm{H}-\mathrm{H}} 8.0 \mathrm{~Hz}, \mathrm{CH}, \mathrm{Tol}\right), 7.78$ (d, $\left.{ }^{3} J_{\mathrm{H}-\mathrm{H}} 8.0 \mathrm{~Hz}, \mathrm{CH}, \mathrm{Tol}\right)$.

2,2,3-Trimethyl-N-tosylaziridine. Colorless crystals, m.p. $91-92^{\circ} \mathrm{C}$. Isolated yield $90 \% .{ }^{1} \mathrm{H} \mathrm{NMR}$ $\left(\mathrm{CDCl}_{3}, 22^{\circ} \mathrm{C}\right.$, Fig. 1), $\delta: 1.11\left(\mathrm{~d},{ }^{3} \mathrm{~J}_{\mathrm{H}-\mathrm{H}}=5.8 \mathrm{~Hz}, 3 \mathrm{H}, \mathrm{C}(3)-\mathrm{CH}_{3}\right), 1.25\left(\mathrm{~s}, 3 \mathrm{H}, \mathrm{CMe}_{2}\right), 1.68\left(\mathrm{~s}, 3 \mathrm{H}, \mathrm{CMe}_{2}\right)$, $2.40(\mathrm{~s}, 3 \mathrm{H}, \mathrm{Me}, \mathrm{Tol}), 2.93\left(\mathrm{~d},{ }^{3} J_{\mathrm{H}-\mathrm{H}}=5.8 \mathrm{~Hz}, 1 \mathrm{H}, \mathrm{C}(3)-\mathrm{H}\right), 7.27\left(\mathrm{~d},{ }^{3} J_{\mathrm{H}-\mathrm{H}}=8.4 \mathrm{~Hz}, 2 \mathrm{H}, \mathrm{CH}, \mathrm{Tol}\right), 7.78(\mathrm{~d}$, $\left.{ }^{3} J_{\mathrm{H}-\mathrm{H}}=8.4 \mathrm{~Hz}, 2 \mathrm{H}, \mathrm{CH}, \mathrm{Tol}\right) ;{ }^{13} \mathrm{C} \mathrm{NMR}\left(\mathrm{CDCl}_{3}, 22{ }^{\circ} \mathrm{C}, \mathrm{Fig} .2\right), \delta: 13.07\left(\mathrm{C}(3)-\mathrm{CH}_{3}\right), 21.09,21.33$ (C(2)Me $\mathrm{M}_{2}$ ), 21.76 (Me, Tol), 48.11 (C(3)), 51.98 (C(2)), 127.14, 129.66 (ortho-, meta-C, Tol), 138.93, 143.65 (ipso-, para-C, Tol). 


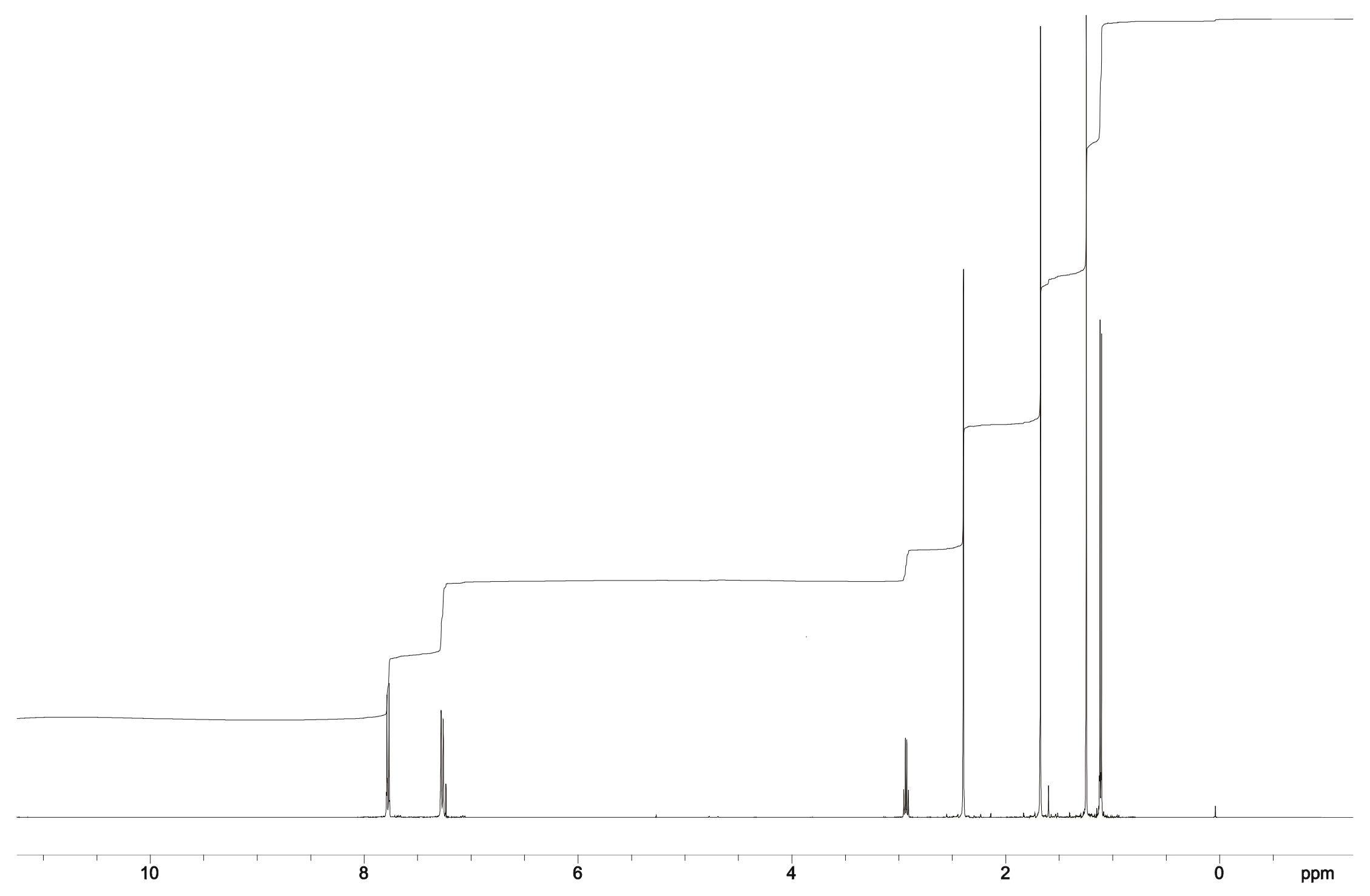

Fig. 1. ${ }^{1} \mathrm{H}$ NMR spectrum of 2,2,3-trimethyl-N-tosylaziridine; $\mathrm{CDCl}_{3}$, r.t. 


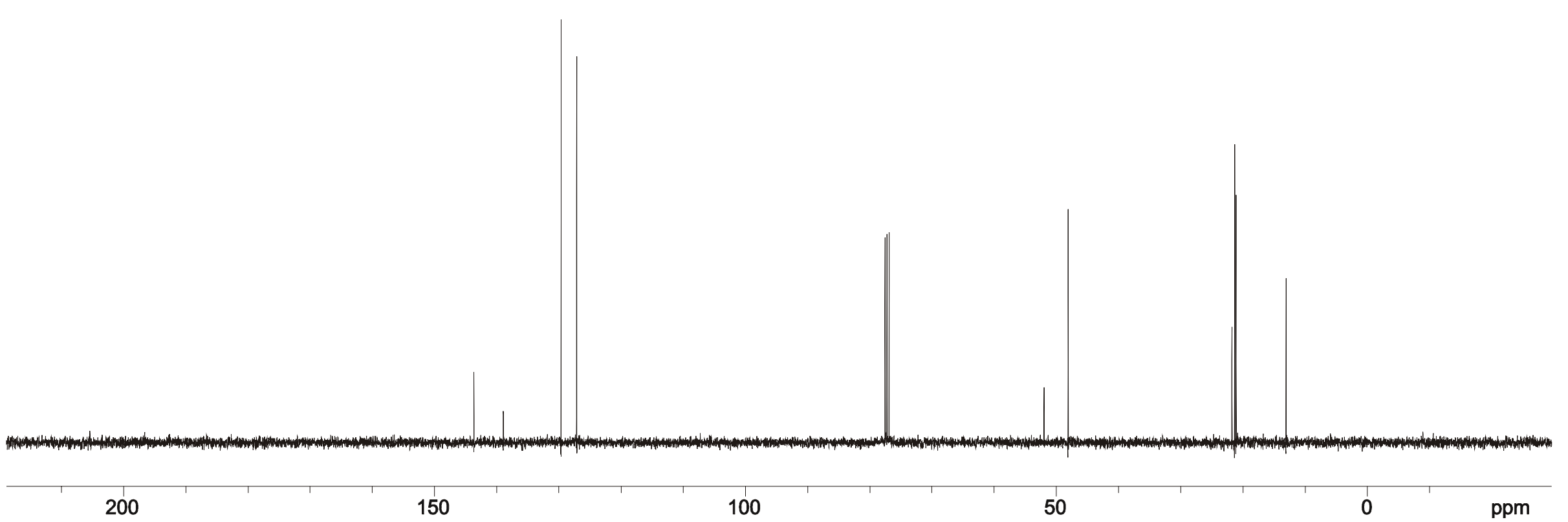

Fig. $2 .{ }^{13} \mathrm{C}$ NMR spectrum of 2,2,3-trimethyl-N-tosylaziridine; $\mathrm{CDCl}_{3}$, r.t. 
Reaction of $\mathrm{LCuBAr}_{4}^{\mathrm{F}}$ with PhINTs.

A solution of $20 \mu \mathrm{mol}$ of $\mathrm{LCuBAr}_{4}^{\mathrm{F}}$ in $1.0 \mathrm{ml}$ of $\mathrm{CD}_{2} \mathrm{Cl}_{2}$ was prepared as described above and removed from a glove box. The solution was cooled to $-60^{\circ} \mathrm{C}$ with an acetone-liquid nitrogen bath and $3.7 \mathrm{mg}$ of PhINTs $(10 \mu \mathrm{mol})$ was added with stirring. In few seconds the mixture turned dark purple. According to NMR data, yield of $(\mathrm{LCu})_{2} \mathrm{NTs}\left(\mathrm{BAr}_{4}\right)_{2}$ was almost quantitative. If 20 $\mu \mathrm{mol}$ of PhINTs were used, the same product was obtained but in lower $(60 \%)$ yield. The compound is stable at room temperature for at least several hours.

${ }^{1} \mathrm{H}$ NMR $\left(\mathrm{CD}_{2} \mathrm{Cl}_{2},-45^{\circ} \mathrm{C}\right), 2.95\left(\mathrm{~s}, 3 \mathrm{H}, \mathrm{CH}_{3}\right), 3.46\left(\mathrm{br} \mathrm{m}, 4 \mathrm{H}, \mathrm{C}_{2} \mathrm{H}_{4}\right), 3.54(\mathrm{~d}, J=14.2 \mathrm{~Hz}$, $4 \mathrm{H}, \mathrm{CH}_{2}$ ), 3.83 (br m, 4H, $\mathrm{C}_{2} \mathrm{H}_{4}$ ), 4.34 (d, J=14.2 Hz, 4H, $\left.\mathrm{CH}_{2}\right), 6.79$ (d, J=7.8 Hz, 2H, CH, Tol), 7.06 (d, J=7.8 Hz, 2H, CH, Tol), 7.51 (br s, $8 \mathrm{H}$, para- $\mathrm{CH}, \mathrm{BAr}_{4}^{\mathrm{F}}$ ), 7.72 (br s, 16H, ortho$\left.\mathrm{CH}, \mathrm{BAr}^{\mathrm{F}}{ }_{4}\right), 8.02(\mathrm{t}, J=7.8 \mathrm{~Hz}, 4 \mathrm{H}$, para- $\mathrm{CH}), 8.17(\mathrm{t}, J=7.8 \mathrm{~Hz}, 2 \mathrm{H}$, para $-\mathrm{CH}), 8.25(\mathrm{~d}, J=$ $7.8 \mathrm{~Hz}, 4 \mathrm{H}$, meta-CH), $8.63(\mathrm{~d}, J=7.8 \mathrm{~Hz}, 4 \mathrm{H}$, meta-CH), 8.81 (d, J=7.8 Hz, 4H, meta-CH).

${ }^{13} \mathrm{C} \mathrm{NMR}\left(\mathrm{CD}_{2} \mathrm{Cl}_{2},-45^{\circ} \mathrm{C}\right), 19.94\left(\mathrm{CH}_{3}\right), 40.38\left(\mathrm{CH}_{2}\right), 46.33\left(\mathrm{C}_{2} \mathrm{H}_{4}\right), 117.73(\mathrm{~m}$, para- $\mathrm{C}$, $\mathrm{BAr}_{4}{ }_{4}$ ), 124.66 (quart, ${ }^{1} J_{\mathrm{C}-\mathrm{F}}=272.7 \mathrm{~Hz}, \mathrm{CF}_{3}, \mathrm{BAr}^{\mathrm{F}}$ ), 127.46, 129.70 (ortho-C, meta-C, Tol), 128.88 (quart, ${ }^{2} J_{\mathrm{C}-\mathrm{F}}=31.7 \mathrm{~Hz}$, meta-C, $\mathrm{BAr}^{\mathrm{F}}{ }_{4}$ ), 134.89 (br s, ortho-C, $\left.\mathrm{BAr}^{\mathrm{F}}{ }_{4}\right), 135.49,136.42$

(meta-C, py), 138.90 (para-C, py), 139.31 (meta-C, py), 139.44 (para-C, py), 142.84, 147.57 (ipso-C, para-C, Tol), 151.29, 155.59 (ortho-C, py), 161.92 (quart, ${ }^{1} \mathrm{~J}_{\mathrm{B}-\mathrm{C}}=50 \mathrm{~Hz}, \mathrm{~B}-\mathrm{C}, \mathrm{BAr}^{\mathrm{F}}{ }_{4}$ ), 165.22 (ortho-C, py). 


\section{References}

(1) Vedernikov, A.N.; Huffman, J.C.; Caulton, K.G. Inorg. Chem. 2002, 41, 6867.

(2) Vedernikov, A.N.; Huffman, J.C.; Caulton, K.G. Inorg. Chem. 2002, 41, 6244.

(3) Parr, R.G.; Yang, W. Density-functional theory of atoms and molecules; Oxford University Press: Oxford, 1989.

(4) Perdew, J.P.; Burke, K.; Ernzerhof, M. Phys. Rev. Lett 1996, 77, 3865.

(5) Ustynyuk, Y. A.; Ustynyuk, L. Y.; Laikov, D.N.; Lunin, V.V. J. Organomet. Chem. 2000, 597, 182.

(6) Stevens, W.J.; Basch, H.; Krauss, M., J. Chem. Phys., 1984, 81, 6026; Stevens, W.J.; Basch, H.; Krauss, M.; Jasien, P., Can. J. Chem., 1992, 70, 612; Cundari, T.R.; Stevens, W.J., J. Chem. Phys., 1993, 98, 5555.

(7) Martin, A.E.; Ford, T.M.; Bulkowski, J.E. J. Org. Chem., 1982, 47, 412.

(8) Nadir, U.K.; Sharma, R.L; Koul, V.K. J. Chem. Soc., Perkin Trans. 1, 1991, 2015.

(9) Bergmeier, S.C.; Seth, P.P. J. Org. Chem., 1997, 62, 2671.

(10) Aggarwal, V.K.; Stenson, R.A.; Jones, R.V.H.; Fieldhouse, R.; Blacker, J. Tetr. Lett., 2001, 42, 1587.

(11) Sugihara, Y.; Iimura, S.; Nakayama, J. Chem. Comm., 2002, 134.

(12) Evans, D.A.; Faul, M.M.; Bilodeau, M.T. J. Am. Chem. Soc., 1994, 116, 2742.

(13) Burgaud, B.G.M.; Horwell, D.C.; Padova, A.; Pritchard, M.C. Tetrahedron, 1996, 52, 13035.

(14) Hegedus, L.S.; McKearin, J.M. J. Am. Chem. Soc., 1982, 104, 2444.

(15) Chanda, B.M.; Vyas, R.; Bedekar, A.V. J.Org. Chem., 2001, 31, 30.

(16) Kim, D.Y.; Kim, H.S.; Choi, Y.J.; Mang, J.Y.; Lee, K. Synth. Comm., 2001, 31, 2463.

(17) Lorne, R.; Julia, S.A. Bull. Soc. Chim. France, 1986, 2, 317.

(18) Ghirardelli, R.; Lucas, H.J. J. Am. Chem. Soc., 1957, 79, 734. 